Full Research Paper

\title{
Gold Nanoparticles With Special Shapes: Controlled Synthesis, Surface-enhanced Raman Scattering, and The Application in Biodetection
}

\author{
Jianqiang Hu, Zhouping Wang and Jinghong $\mathrm{Li}^{*}$ \\ Department of Chemistry, Key Laboratory of Bioorganic Phosphorus Chemistry \& Chemical Biology, \\ Tsinghua University, Beijing 100084, China \\ E-mail: jhli@mail.tsinghua.edu.cn
}

* Author to whom correspondence should be addressed. Tel \& Fax: (+86)-10-62795290

Received: 12 November 2007 / Accepted: 12 December 2007 / Published: 14 December 2007

\begin{abstract}
Specially shaped gold nanoparticles have intrigued considerable attention because they usually possess high-sensitivity surface-enhanced Raman scattering (SERS) and thus result in large advantages in trace biodetermination. In this article, starch-capped gold nanoparticles with hexagon and boot shapes were prepared through using a nontoxic and biologically benign aqueous-phase synthetic route. Shape effects of gold nanoparticles on SERS properties were mainly investigated, and found that different-shaped gold nanoparticles possess different SERS properties. Especially, the boot-shaped nanoparticles could induce more 100-fold SERS enhancements in sensitivity as compared with those from gold nanospheres. The extremely strong SERS properties of gold nanoboots have been successfully applied to the detection of avidin. The unique nanoboots with high-sensitivity SERS properties are also expected to find use in many other fields such as biolabel, bioassay, biodiagnosis, and even clinical diagnosis and therapy.
\end{abstract}

Keywords: Gold nanoparticle, Special shape, Surface-enhanced Raman scattering, Biodetection 


\section{Introduction}

Nano-building blocks with novel shapes exhibit more unique physical and chemical properties in comparison with nanostructures with common shapes (e.g., sphere or rod) since intrinsic properties of metallic nanostructures depend vitally on particle shape [1-3]. Their displaying novel properties have wider and more effective biological and medical applications, for example, these building blocks have potential for fabricating biological labels, biological sensors, bioanalysis and biodiagnosis technologies, diagnosis and monitoring of diseases, drug discovery, environmental detection of biological reagents, and even medical clinical diagnosis and therapy [1, 4-7]. Two major challenges for real-time determination of biomolecules are of key importance: (i) biocompatibility and (ii) high sensitivity in bioanalysis. Achieving an exciting substrate with very high sensitivity plays a pivotal role in biological assays, for example, the urgent need for measuring disease diagnosis markers that present at ultralow levels during early stages of disease progress. This problem can be solved through using polymerase chain reaction (PCR) amplification. However, it is, to some extent, restricted due to its complexity, potential contamination, and cost [8]. Thereby, it is a great challenge to effectively monitor biomolecular interactions in the absence of PCR-like amplification protocols. Nevertheless, nanotechnology offers unique opportunities and good platforms for creating highly sensitive biodetecting devices and ultrasensitive bioassays because nano-building blocks, especially novelshaped nanoparticles, exhibit unique physical and chemical properties $[2,9,10]$.

Bio-detection sensitivity of nanomaterials associates intimately with their physical and chemical properties depending on the component, size, and shape [11-16]. Recent years, nanoparticles with different components and dimensions have been widely applied to detect biological molecules. For example, Keating's group designed a new approach for the detection of DNA hybridization based on nanoparticle-amplified surface plasmon resonance (SPR), using which a more than 1000-fold improvement in sensitivity was obtained for the target oligonucleotide as compared with the unamplified binding event [12]. Nie and his co-workers used colloidal gold nanocrystals to recognize and detect specific DNA sequences and single-base mutations in a homogeneous format [13]. Silica nanoparticles bioconjugated with fluorescent dye were also used to perform a rapid bioassay for single bacterial cell [14]. Recent work in this field was the successful fabrication of a label-free biochip based on noble metal nanoparticles, demonstrating that the sizes of gold nanoparticles with diameters in the range of 12-48 nm significantly affect its sensitivity [15]. For example, the detection limit for streptavidin-biotin binding of a biochip fabricated from 39-nm-diameter nanoparticles was 20-fold better than a previously reported biochip fabricated from 13-nm-diameter gold nanoparticles. Among all nanoparticles, gold nanoparticles as bio-detection precursor should be predominantly interesting because it exhibits the best compatibility with biomolecules. But, bio-detection sensitivity derived from spherical nanoparticles isn't still strong enough to achieve the real-time determination of trace biomolecules and the interaction between biomolecules. Nevertheless, it is reasonable to infer that novel shape nanoparticles might be hopeful to reach this aim because their displaying novel properties may greatly improve biological detection sensitivity [16].

In this article, we report the synthesis of starch-capped gold nanoparticles with hexagon and boot shapes via designing a biologically benign synthetic strategy and their shapes can be controlled through varying D-glucose concentration. In this process, the nanoparticles were prepared by the 
reduction of chloroauric acid $\left(\mathrm{HAuCl}_{4}\right)$ with $\mathrm{D}$-glucose in the presence of starch and water respectively served as a biologically benign capping agent and solvent. These starch-capped gold nanoparticles are nontoxicity for biological body and good biocompatibility because the nanoparticle toxicity mainly depends on its capping agent but not nanoparticles itself [17]. We subsequently studied shape effects of metal nanoparticles on SERS properties through using differently shaped gold nanoparticles respectively served as SERS carriers, and found that gold nanoparticles with the boot shape could induce ultrasensitive SERS signals, using which the detections of avidin were successfully acquired.

\section{Results and Discussion}

\subsection{TEM and HRTEM characterization of Au colloids with hexagon and boot shapes}

Our synthesis was performed by the reduction of $\mathrm{HAuCl}_{4}$ with $\mathrm{D}$-glucose in the presence of starch. Fig. 1A and 1B show typical TEM images of differently shaped gold nanoparticles prepared using the present method. Hexagon-shaped gold nanoparticles synthesized using $0.1 \mathrm{mM}$ D-glucose have the side length of $12 \pm 2 \mathrm{~nm}$. A decrease in the concentration $(0.02 \mathrm{mM})$ of D-glucose changes gold nanoparticles' shape into a boot shape. Fig. 1B shows the representative TEM image of gold nanoboots, which have the length of $56 \pm 9 \mathrm{~nm}$ and the narrowing width from $23 \pm 5$ to $16 \pm 3 \mathrm{~nm}$ along its longitudinal axis. Their insets are the corresponding TEM images of single magnified gold nanoparticles whose sizes and shapes could be clearly seen, respectively. HRTEM images of the gold nanohexagons and nanoboots are given in Fig. 2, in which the lattice fringes of the gold nanohexagons and nanoboots are respectively visible (20). XRD and EDX spectrum measurements confirm that the crystal structures of the nanohexagons or nanoboots are face-center-cubic (fcc) (Joint Committee for Powder Diffraction Standards (JCPDS), File No. 4-0783) and the samples are pure Au.

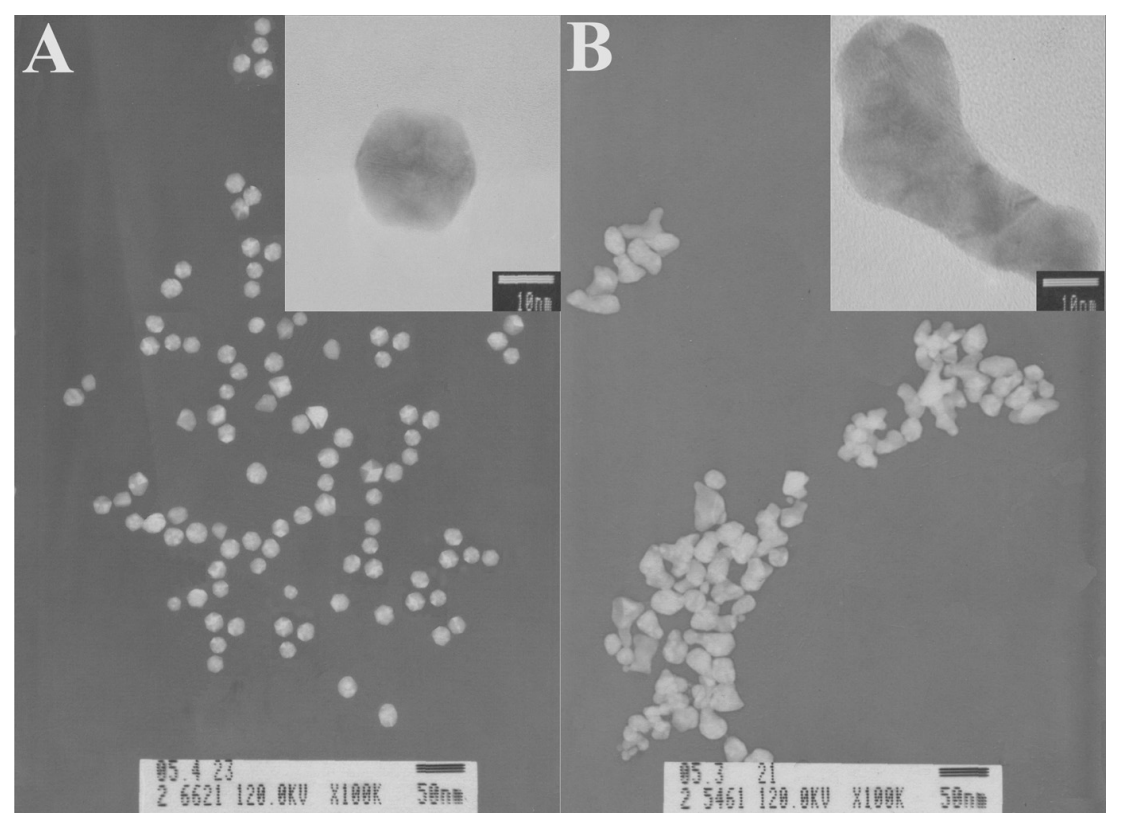

Figure 1. TEM images of (A) hexagon- and (B) boot-shaped gold nanoparticles prepared using the present synthetic route. Their insets are the corresponding TEM images of single gold nanoparticles, respectively. Scale bars: $50 \mathrm{~nm}$ and $10 \mathrm{~nm}$ (inset). 


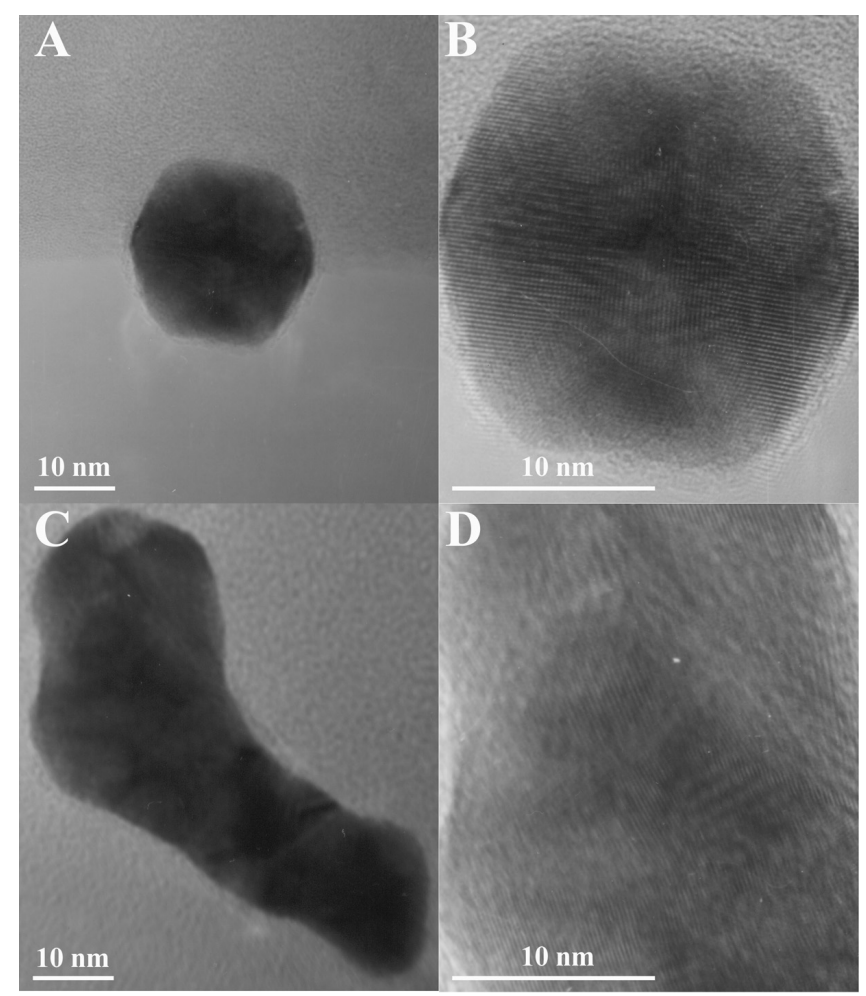

Figure 2. (A, C) TEM and (B, D) HRTEM images of the gold nanohexagons and nanoboots synthesized respectively using 0.1 and $0.02 \mathrm{mM}$ D-glucose.

Moreover, XRD pattern of the gold sample with the boot shape synthesized using $0.02 \mathrm{mM}$ Dglucose, indicating that crystal structure of the nanoboots was fcc (JCPDS 4-0783). Another sample with hexagon shape had the same result. EDX spectra of the gold nanohexagons (A) and nanoboots (B) synthesized respectively using 0.1 and $0.02 \mathrm{mM}$ D-glucose, indicating that the samples were pure Au.
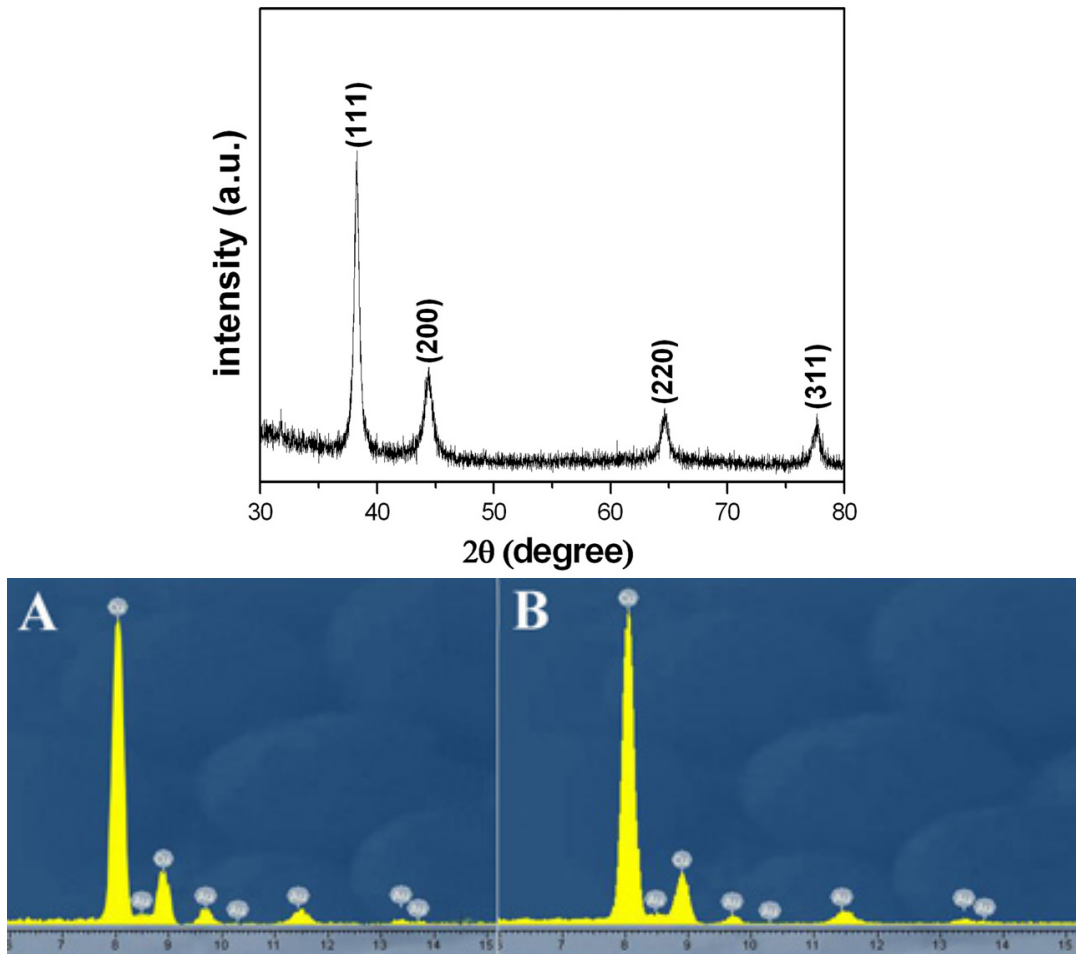

Figure 3. XRD pattern of gold nanoboots and EDX spectra of gold nanohexagons (A) and nanoboots (B). 


\subsection{UV-visible absorption property of different-shaped gold nanoparticles}

Novel properties derived from unusually shaped nanoparticles can be generally exhibited through plasmon absorption spectroscopy because their optical properties of aqueous suspensions intimately associate with the shape $[1,3,16,18,19]$. For example, the UV-visible spectrum for the colloidal solution of spherical gold nanoparticles prepared using the present method shows a narrow peak at approximately $523 \mathrm{~nm}$ (Fig. 4, curve a). Compared with the spherical nanoparticles, the UV-visible absorption of uniquely shaped gold nanoparticles usually shows the red-shift and wider peak [16, 18]. Curves $b$ and $d$ of Fig. 4 give UV-visible absorption spectra taken from the final reaction solutions synthesized using 0.1 and $0.02 \mathrm{mM}$ D-glucose, respectively, whose colors are respectively violet red and light violet (the inset of Fig. 4). The nanohexagon colloidal solution has an absorption band at about $550 \mathrm{~nm}$ with the full width at half-maximum (FWHM) of ca. $65 \mathrm{~nm}$ (Fig. 4, curve b). In comparison, the nanoboot solution shows, similar to the reported gold nanotadpoles (Fig. 4, curve c) [18], a rather broader peak at about $545 \mathrm{~nm}$ with FWHM of ca. $145 \mathrm{~nm}$ (Fig. 4, curve d). It can be clearly seen from Fig. 4 that the more irregular nanoparticles possess the more red-shifts and wider absorption peaks, indicating that the UV-visible absorption property of colloidal solution intimately depends on its shape. These broad and multiple absorption peaks should probably result from the variable dimensions along to multiple axes of these particles [16].



Figure 4. UV-visible absorption spectra of sphere-shaped (a), hexagon-shaped (b), tadpole-shaped (c), and boot-shaped (d) gold nanoparticles taken respectively from their corresponding colloidal solutions, and the inset is digital photos of the reaction mixtures of the nanoparticles with hexagon and boot shapes, respectively.

\subsection{SERS property of different-shaped gold nanoparticles}

SERS is an attractive and promising analytical tool for real-time detection of biomolecules due to its ultrahigh sensitivity [20, 21]. The sensitivity of SERS obtained from noble metal nanoparticles strongly depends on the size and shape, especially the latter $[16,22]$. In the nineties of the twentieth century, Nie's group demonstrated size-dependent SERS enhancement in single metal nanoparticles 
[23]. Suzuki and his co-workers also reported that gold nanoparticle films with different sizes generate different-intensity SERS signals [24]. Spherical gold nanoparticles served as SERS-active substrate usually give an enhancement in the order of from 103 to 106. However, the SERS sensitivity from spherical gold nanoparticles is still insufficient to detect trace biomolecules and/or the interaction between biomolecules, which may be overcome through using novel shape nanomaterials served as SERS substrate for the improvement of bio-detection limit [25, 26]. Fig. 5 shows Raman spectrum of $10 \mathrm{M} \mathrm{SCN}^{-}$solution and SERS spectra of SCN- adsorbed at different-shaped gold nanoparticles. The intensities obtained from the nanoboots and nanotadpoles are about two orders of magnitude stronger than those of the nanohexagones and nanospheres, which are consistent with the previously reported SERS spectra for gold nanoparticles with novel shapes [16]. Furthermore, the boot-induced SERS also shows the stronger intensity than that from the nanotadpoles. It is well known that the lightning effect can result in the largest electric field near the sharpest surface, e.g., at the sharp ends of nanoparticles. As a consequence, the Raman enhancement reaches its maximum value at the sharpest surface [27]. On the basis of the electromagnetic enhancement theory, the SERS intensity induced by metal nanoparticles intimately depends on the total number of the sharp ends. From the spheres, hexagons, and tadpoles to boots, the total sharp numbers gradually increase, the SERS activities dramatically increase, too. Indeed, our experimental results showed in Fig. 5 agree well with this theoretical prediction. And that the different SERS frequencies of SCN- absorbed at different shape gold nanoparticles are due to the different interaction between gold nanoparticles and adsorbed molecule [28]. The stronger the interaction is, the higher the frequency shifts to. Among sphere, hexagon, tadpole, and boot, this means that the boots have the highest SERS frequency, followed by the tadpoles, and the lowest frequency belongs to the spheres and hexagons, indicating that the boots have the most active "hot" areas. The most active area of the nanoboots is further evidence of the strongest SERS and CL signals.
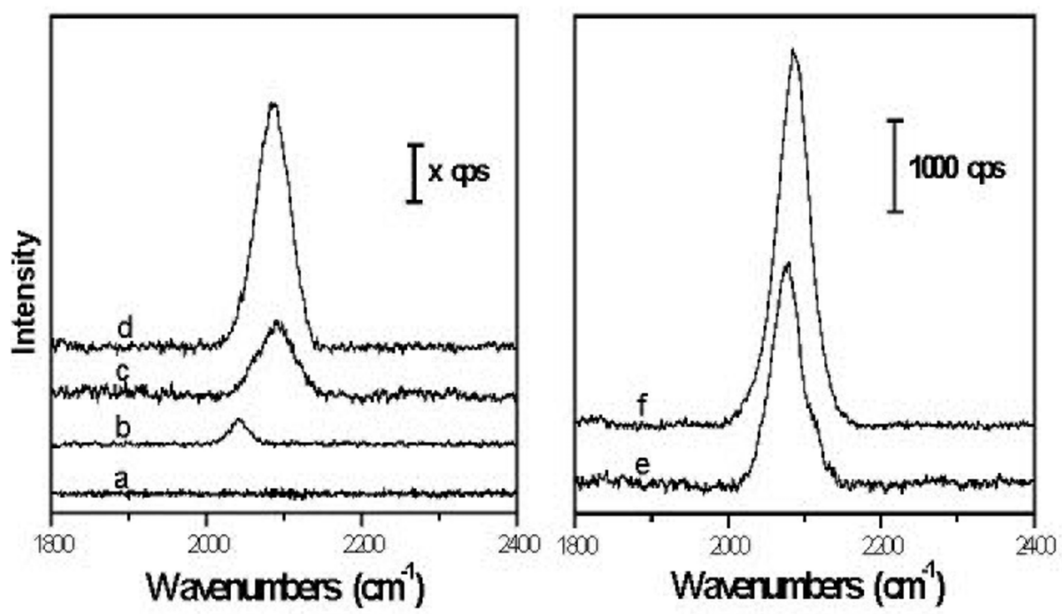

Figure 5. SERS spectrum of (a) blank on ITO glass slice assembled with spherical gold nanoparticles, Raman spectrum of (b) $10 \mathrm{M} \mathrm{SCN}^{-}$solution, and SERS spectra of $\mathrm{SCN}^{-}$on ITO glass slices assembled with (c) spheres, (d) hexagons, (e) tadpoles, and (f) boots. Integration times are (a) $60 \mathrm{~s}$, (b) $800 \mathrm{~s}$ (scale bar: $0.25 \mathrm{cps}$ ), (c) $20 \mathrm{~s}$ (scale bar: $10 \mathrm{cps}$ ), (d) $20 \mathrm{~s}$ (scale bar: $10 \mathrm{cps}$ ), (e) $1 \mathrm{~s}$ (scale bar: 1000 cps), and (f) $1 \mathrm{~s}$ (scale bar: $1000 \mathrm{cps}$ ). 
Table 1. Calculated Surface Enhancement Factors (EF) for $\mathrm{SCN}^{-}$on ITO Glass Slices Assembled with Gold Nanoparticles of Different Shapes

\begin{tabular}{|l|c|}
\hline Nanoparticle shape & EF* $^{*}$ \\
\hline Sphere & $1.26 \pm 0.52 \times 10^{6}$ \\
\hline Hexagon & $4.01 \pm 0.97 \times 10^{6}$ \\
\hline Tadpole & $2.45 \pm 0.23 \times 10^{8}$ \\
\hline Boot & $4.04 \pm 0.33 \times 10^{8}$ \\
\hline
\end{tabular}

*The EFs from the SERS peaks at about $2075 \mathrm{~cm}^{-1}$ of $\mathrm{SCN}^{-}$were calculated using the typical expression of $\left\{\left[\mathrm{I}_{S E R S}\right] \times\left[\mathrm{M}_{\text {bulk }}\right]\right\} /\left\{\left[\mathrm{I}_{\text {Raman }}\right] \times\left[\mathrm{M}_{a d s}\right]\right\}$.

We also calculated surface enhancement factors (EF) of the nanoparticles with different shapes using the classical formula, i.e., $\frac{\left[I_{\mathrm{SERS}}\right] \times\left[M_{\text {bulk }}\right]}{\left[I_{\mathrm{Raman}}\right] \times\left[M_{\mathrm{ads}}\right]}$. Wherein ISERS is the intensity of SCN- in the SERS spectrum, IRaman is the intensity of $\mathrm{SCN}^{-}$in the Raman spectrum, Mbulk is the number of $\mathrm{SCN}^{-}$sampled in the bulk, and Mads is the number of $\mathrm{SCN}^{-}$absorbed and sampled in the SERS substrate. All spectra were standardized for acquisition time. The illuminated focus spot diameter is 2 $\mu \mathrm{m}$, and thus the absorbed total molecule number of about $1.57 \times 10^{-17} \mathrm{~mol}$ in the SERS experiments is calculated by estimating the bonding density of $\mathrm{SCN}^{-}$molecules in a self-assembly monolayer (SAM) [29]. Table 1 lists calculated surface $\mathrm{EFs}$ for $\mathrm{SCN}^{-}$on ITO glass slices assembled with gold nanoparticles of different shapes. The boots induce the largest surface EFs (more than 108), and the spheres generate the smallest enhancement (ca. 106). The strong enhancement for the boots contributes not only to the total number of its sharp ends but also its absorption at $785 \mathrm{~nm}$ that generates effectively plasmon resonance enhancement as compared with the spheres, hexagons, and tadpoles. As a result, the nanoboots could serve as an ideal SERS activity substrate to probe biomolecules because of its enhancement of two orders of magnitude as compared with the nanospheres [30].

\subsection{Real-time determination of avidin by SERS}

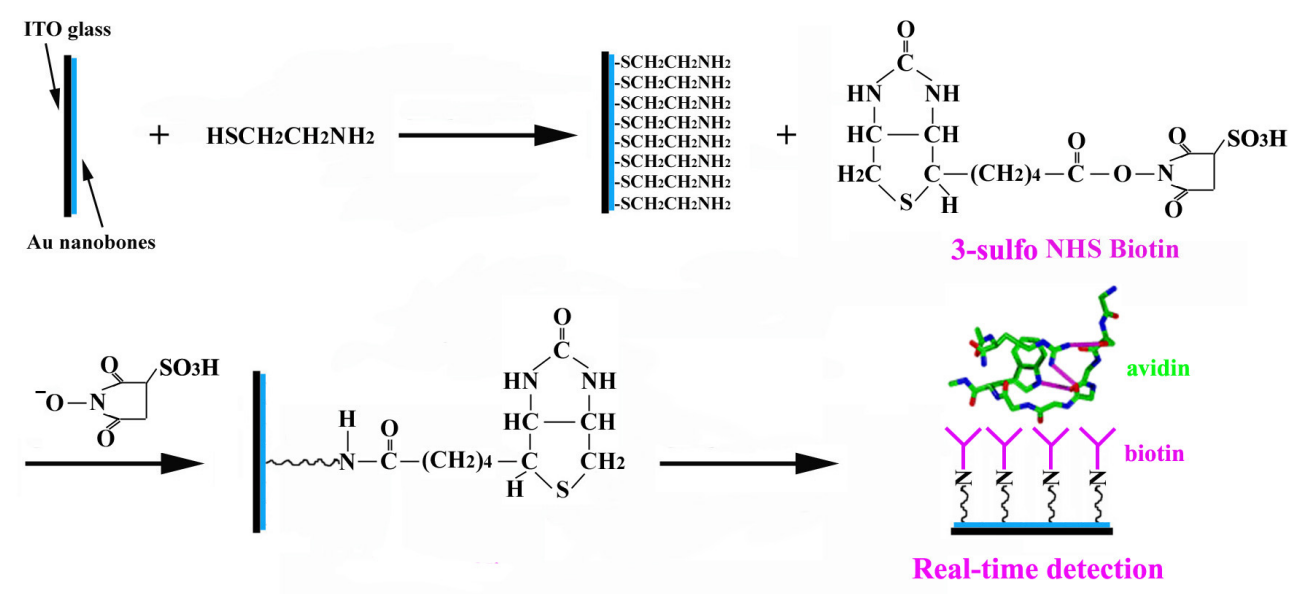

Figure 6. Schematic illustration of the real-time determination of avidin. 


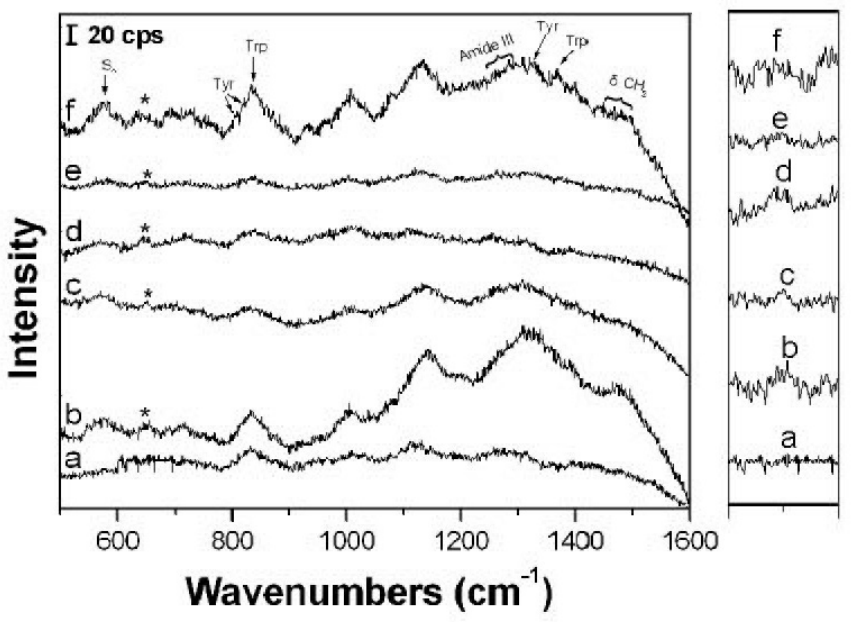

A



B

Figure 7. (A) SERS spectra of (a) the coupling complex between mercaptoethylamine-assembled gold nanoboots and biotins and its real-time detection of (b) $1 \mathrm{mg} / \mathrm{mL}$, (c) $0.1 \mathrm{mg} / \mathrm{mL}$, (d) $0.01 \mathrm{mg} / \mathrm{mL}$, and (e, f) $0.001 \mathrm{mg} / \mathrm{mL}$ avidin. Except curve f (integration time: $50 \mathrm{~s}$; scale bar: $8 \mathrm{cps}$ ), integration times of all SERS spectra were $20 \mathrm{~s}$ (scale bar: $20 \mathrm{cps}$ ). The right plot shows magnified spectrum peaks at 600 $700 \mathrm{~cm}^{-1}$ from the asterisks of the left plot. (B) Plot of SERS intensity respond to the concentration of avidin.

It is important to note that biotin can form a very high binding affinity with avidin, an egg white protein [31-35]. The strong bonding property between biotin and avidin also offers new opportunities for detecting avidin. As described in Section 3.3. and represented in Fig. 6, a self-assembly procedure was employed on the indium tin oxide (ITO) glass slice for SERS determination of avidin. Fig.7 (A) shows SERS spectra of the coupling complex between mercaptoethylamine-assembled gold nanoboots and biotins and its real-time detection of different concentration avidin. All these SERS spectra are reproducible at different sites on a substrate, with a standard deviation of $<20 \%$. The bands (at about $648 \mathrm{~cm}^{-1}$ ) marked with asterisks attribute to the biotin-avidin complex [36]. As the concentration of avidin decreases from $1 \mathrm{mg} / \mathrm{mL}$ (Fig. $7 \mathrm{~b}$ ) to $1 \mu \mathrm{g} / \mathrm{mL}$ (Fig. 7e), the SERS intensities corresponding to the ligand of biotin and avidin progressively decrease, which can be clearly seen from the magnified spectrum peaks at $600-700 \mathrm{~cm}^{-1}$ from the asterisks (the right plot of Fig. 7(A) and (B)). The band obtained using $1 \mu \mathrm{g} / \mathrm{mL}$ avidin is hardly discernable but it can be again identified clearly if longer integration time is used (Fig. 7f). The appearance of the amide III mode in the 1230-1284 $\mathrm{cm}^{-1}$ region and lone disulfide of avidin subunits at about $545 \mathrm{~cm}^{-1}$ further confirm the presence of the biotinavidin interactions. Other bands at about 806, 821, 834, 1323, 1364, and 1440-1480 $\mathrm{cm}^{-1}$ correspond respectively to the hydrogen bonds of biotin and its ligand with avidin, the side-chains of avidin, and methylene of biotin or avidin. These results are in agreement with the reported literatures [36-38], indicating that the boot-shaped gold nanoparticles could effectively and real-time detect avidin molecules down to $0.01 \mathrm{unit} / \mathrm{mL}$ by making use of its high-sensitivity SERS activity. 


\section{Experimental Section}

\subsection{Reagents and materials}

Dihydrate chloroauric acid ( $\left.\mathrm{HAuCl}_{4} \quad 2 \mathrm{H}_{2} \mathrm{O}, 99 \%\right)$, biotin 3-sulfo-N-hydroxysuccinimide (biotin 3sulfo-NHS, 90\%), avidin (from egg white (13 units/mg solid)), glucose oxidase (GOD), mercaptoethylamine (99\%), tetra-ethylorthosilicate (90\%), and sodium dodecylsulfonate (99\%) were purchased from Aldrich and ACROS. Luminol was obtained from Merck (Germany). The following analytical pure reagents were purchased from Tianjin VAS Chemical Reagent Co.: starch, D-glucose, tri-sodium citrate, potassium chloroide, potassium thiocyanate, luminol, glucose oxidase, sodium dihydrogen phosphate, disodium hydrogen phosphate, concentrated hydrochloric acid, absolute acetone, and high pure argon gas. All above reagents were used without further purification. $18 \mathrm{M} \Omega$ $\mathrm{cm}^{-1}$ water was used to prepare all aqueous solutions. All glassware used was washed with aqua regia and rinsed with ultrapure water prior to use. $0.1 \mathrm{M}$ of phosphate buffer (PBS, $\mathrm{pH}=7.0$ ) was made by an equal volume mixture of $0.0780 \mathrm{M}$ sodium dihydrogen phosphate and $0.122 \mathrm{M}$ disodium hydrogen phosphate in an aqueous solution.

\subsection{Preparation of gold nanoparticles with different shapes}

In a typical procedure for the preparation of novel gold nanoparticles with hexagon and boot shapes, two $150 \mathrm{~mL}$ round-bottom flasks containing $50 \mathrm{~mL}$ (final volume) aqueous solution of $0.1 \mathrm{mM}$ $\mathrm{HAuCl}_{4}$ were first prepared, and then added $5 \mathrm{~mL}$ of $0.17 \%$ starch (wt $\%$ ), respectively. The two solutions were purged oxygen with argon for $10 \mathrm{~min}$, respectively. Next, $5 \mathrm{~mL}$ and $1 \mathrm{~mL}$ of $1 \mathrm{mM} \mathrm{D-}$ glucose purged with argon were introduced to the as-prepared solutions, respectively. Finally, each reaction solution kept stirring at $40{ }^{\circ} \mathrm{C}$ for $24 \mathrm{~h}$. The two reaction mixtures finally turned turbid with violet red and light violet, respectively. Gold nanoparticles with sphere and tadpole shapes were synthesized according to our previously synthetic procedure with a little modification [18]. The average diameter of the gold nanospheres was around $22 \mathrm{~nm}$; for the gold nanotadpoles, their average length, maximal width, and maximal height were respectively ca. $82 \mathrm{~nm}, 21 \mathrm{~nm}$, and $6.9 \mathrm{~nm}$.

\subsection{Real-time determination of avidin molecules}

First, the coupling complex between assembled gold nanoboots and biotins for the determination of avidin was fabricated according to the following procedure (shown in Fig. 6): (i) cleaned indium tin oxide (ITO) glass slice was placed in boot-shaped gold colloid to allow them self-assemble, and the slice was removed after $24 \mathrm{~h}$ and blew them with $\mathrm{N}_{2}$. (ii) To form amine-functionalized group on the nanoparticle surfaces, the Au-assembled ITO reacting with $10 \mathrm{mM}$ mercaptoethylamine was similarly taken out after $6 \mathrm{~h}$ and washed with $18 \mathrm{M} \Omega \mathrm{cm}^{-1}$ water and dried with $\mathrm{N}_{2}$. (iii) The aminefunctionalized Au-ITO slice then reacted with $0.1 \mathrm{mg} / \mathrm{mL}$ biotin 3-sulfo-N-hydroxysuccinimide in 0.1 M PBS (pH 7.0), and was washed and dried with the same procedure. Next, a droplet (approximately $50 \mu \mathrm{L}$ ) of $1000,100,10$, and $1 \mu \mathrm{g} / \mathrm{mL}$ avidin solutions was pipetted respectively onto as-prepared dried bio-complexes and immediately detected after $2 \mathrm{~min}$. The $785 \mathrm{~nm}$ laser was focused, via a $20 \times$ 0.4 NA objective lens, through the sample solution and onto the bio-complex interface by adjusting the 
microscope stage. Finally, spectrum acquisition was started immediately. Laser intensity at the focal spot was about $1.2 \mathrm{~mW} / \mathrm{cm}$. The focus spot size was $5 \mu \mathrm{m}$, the spectral resolution was $1 \mathrm{~cm}^{-1}$, and an integration time was $60 \mathrm{~s}$.

\subsection{Instruments}

Transmission electron microscopic (TEM) measurement was performed with a Hitachi Model H800 microscope operated at $100 \mathrm{kV}$. High-resolution TEM (HRTEM) images and energy-dispersive Xray (EDX) spectra were obtained on a JEOL JEM-2010F microscope operated at $200 \mathrm{kV}$. UV-visible spectra were acquired using a Shimadzu UV-21005 spectrophotometer using two $1 \mathrm{~cm}$ quartz cells. Xray diffraction (XRD) pattern was recorded on a powder sample using a Bruker D8 Advance X-ray diffractometer with $\mathrm{Cu}_{\mathrm{K} \alpha}$ radiation $(\lambda=1.5418 \AA)$. Raman and surface-enhanced Raman scattering (SERS) spectra were obtained with a microscopic confocal Raman spectrometer (Renishaw, RM 2000) operated with a semiconductor laser $(785 \mathrm{~nm})$, laser intensity (about $0.47 \mathrm{~W} / \mathrm{cm}$, and objective lens $(50$ $\mathrm{X})$.

\section{Conclusion}

In summary, we demonstrated that starch-capped gold nanoparticles with novel and controllable shapes (e.g., boot and hexagon) could be synthesized using a biologically benign synthetic route. Differently shaped gold nanoparticles could excite or induce to give rise to different SERS properties through surface catalysis or electromagnetic field, respectively. Along with the shape change from sphere, hexagon, and tadpole to boot, SERS intensities induced gradually increase. In comparison with the spherical nanoparticles, the boot-shaped nanoparticles could generate the SERS enhancements of more two orders of magnitude. The extremely strong SERS properties induced by the nanoboots have been successfully utilized to probe biomolecules through achieving the success of the coupling between biomolecules and gold nanoboots. To determinate the bio-detection sensitivity of the coupling complex, we selected a representatively probed biomolecule, i.e., avidin. It was found that we could effectively and real-time detect the concentration of avidin down to $0.01 \mathrm{unit} / \mathrm{mL}$. The nanoboots with high-sensitivity SERS properties could be also utilized to detect many other biomolecules and are expected to find use in many fields such as biolabel, bioassay, biodiagnosis, and even clinical diagnosis and therapy.

\section{Acknowledgements}

This work was supported financially by the National Natural Science Foundation of China (No. 20435010), National Basic Research Program of China (No. 2007CB310500). We also acknowledged the financial support from Postdoctoral Science Foundation of China (023205035, 2005038302). 


\section{References and Notes}

1. Jin, R. C.; Cao, Y. W.; Mirkin, C. A.; Kelly, K. L.; Schatz G. C.; Zheng J. G. Photoinduced conversion of silver nanospheres to nanoprisms. Science 2001, 294, 1901-1903.

2. El-Sayed M. A. Some interesting properties of metals confined in time and nanometer space of different shapes. Acc. Chem. Res. 2001, 34, 257-264.

3. Wang, Z. P.; Hu, J. Q.; Jin, Y.; Yao, X.; Li, J. H. In situ amplified chemiluminescent detection of DNA and immunoassay of IgG using special-shaped gold nanoparticles as label. Clin. Chem. 2006, 52, 1958-1961.

4. Yu, L.; Banerjee, I. A.; Matsui, H. Direct growth of shape-controlled nanocrystals on nanotubes via biological recognition. J. Am. Chem. Soc. 2003, 125, 14837-14840.

5. Penn, S. G.; He, L.; Natan, M. J. Nanoparticles for bioanalysis. Curr. Opin. Chem. Biol. 2003, 7, 609-615.

6. Turner, A. P. F. Biosensors--sense and sensitivity. Science 2000, 290, 1315-1317.

7. Haes, A. J.; Van Duyne, R. P. A nanoscale optical biosensor: sensitivity and selectivity of an approach based on the localized surface plasmon resonance spectroscopy of triangular silver nanoparticles. J. Am. Chem. Soc. 2002, 124, 10596-10604.

8. Wang, J. Nanomaterial-based amplified transduction of biomolecular interactions. Small 2005, 1, 1036-1043.

9. Deng, F. J.; Yang,Y. Y.; Hwang, S.; Shon, Y. S.; Chen, S. W. Fullerene-functionalized gold nanoparticles: electrochemical and spectroscopic properties. Anal. Chem. 2004, 76, 6102-6107.

10. Tsunoyama, H.; Sakurai, H.; Negishi, Y.; Tsukuda, T. Size-specific catalytic activity of polymerstabilized gold nanoclusters for aerobic alcohol oxidation in water. J. Am. Chem. Soc. 2005, 127, 9374-9375.

11. Hu, J. Q.; Wen, Z. H.; Wang, Q.; Yao, X.; Zhang, Q.; Zhou, J. H.; Li, J. H. Controllable synthesis and enhanced electrochemical properties of multifunctional Aucore $\mathrm{Co}_{3} \mathrm{O}_{4}$ shell nanocubes. $J$. Phys. Chem. B 2006, 110, 24305-24310.

12. He, L.; Musick, M. D.; Nicewarner, S. R.; Salinas, F. G.; Benkovic, S. J.; Natan M. J.; Keating, C. D. Colloidal Au-enhanced surface plasmon resonance for ultrasensitive detection of DNA hybridization. J. Am. Chem. Soc. 2000, 122, 9071-9077.

13. Maxwell, D. J.; Taylor, J. R.; Nie, S. M. Self-assembled nanoparticle probes for recognition and detection of biomolecules. J. Am. Chem. Soc. 2002, 124, 9606-9612.

14. Zhao, X. J.; Hilliard, L. R.; John Mechery, S.; Wang, Y. P.; Bagwe, R. P.; Jin, S. G.; Tan, W. H. A rapid bioassay for single bacterial cell quantitation using bioconjugated nanoparticles. P. Natl. Acad. Sci. USA 2004, 101, 15027-15032.

15. Nath, N.; Chilkoti, A. Label-free biosensing by surface plasmon resonance of nanoparticles on glass: optimization of nanoparticle size. Anal. Chem. 2004, 76, 5370-5378.

16. Orendorff, C. J.; Gole, A.; Sau, T. K.; Murphy, C. J. Surface-enhanced Raman spectroscopy of self-assembled monolayers: sandwich architecture and nanoparticle shape dependence. Anal. Chem. 2005, 77, 3261-3266.

17. Derfus, A. M.; Chan, W. C. W.; Bhatia, S. N. Probing the cytotoxicity of semiconductor quantum dots. Nano Lett. 2004, 4, 11-18. 
18. Hu, J. Q.; Zhang, Y.; Liu, B.; Liu, J. X.; Zhou, H. H.; Xu, Y. F.; Jiang, Y. X.; Yang, Z. L.; Tian, Z. Q. Synthesis and properties of tadpole-shaped gold nanoparticles. J. Am. Chem. Soc. 2004, 26, 9470-9471.

19. Link, S.; El-sayed, M. A. Spectral properties and relaxation dynamics of surface plasmon electronic oscillations in gold and silver nanodots and nanorods. J. Phys. Chem. B 1999, 103, 8410-8426.

20. O'neal, P. D.; Cote, G. L.; Motamedi, M.; Chen J.; Lin, W. C. Feasibility study using surfaceenhanced Raman spectroscopy for the quantitative detection of excitatory amino acids (vol 8, pg 33, 2003). J. Biomed. Opt. 2003, 8, 316-316.

21. Sulk, R.; Chan, C.; Guicheteau, J.; Gomez, C.; Heyns, J. B. B.; Corcoran, R.; Carron, K. Surfaceenhanced Raman assays (SERA): Measurement of bilirubin and salicylate. J. Raman. Spectrosc. 1999, 30, 853-859.

22. Hu, J. Q.; Chen, Q.; Xie, Z. X.; Han, G. B.; Wang, R. H.; Ren, B.; Zhang, Y.; Yang, Z. L.; Tian, Z. Q. A simple and effective route for the synthesis of crystalline silver nanorods and nanowires. Adv. Funct. Mater. 2004, 14, 183-189.

23. Emory, S. R.; Haskins, W. E.; Nie, S. M. Direct observation of size-dependent optical enhancement in single metal nanoparticles. J. Am. Chem. Soc. 1998, 120, 8009-8010.

24. Suzuki, M.; Niidome, Y.; Kuwahara, Y.; Terasaki, N.; Inoue, K.; Yamada, S. Surface-enhanced nonresonance Raman scattering from size- and morphology-controlled gold nanoparticle films. $J$. Phys. Chem. B 2004, 108, 11660-11665.

25. Sharma, R. K.; Sharma, P.; Maitra, A. J. Size-dependent catalytic behavior of platinum nanoparticles on the hexacyanoferrate(III)/thiosulfate redox reaction. J. Colloid. Interface Sci. 2003, 265, 134-140.

26. Zhang, Y.; Gu, C.; Schwartzberg, A. M.; Zhang, J. Z. Surface-enhanced Raman scattering sensor based on D-shaped fiber. Appl. Phys. Lett. 2005, 87, Art. No. 123105.

27. Gersten, J.; Nitzan, A. Electromagnetic theory of enhanced Raman scattering by molecules adsorbed on rough surfaces. J. Chem. Phys. 1980, 73, 3023-3037.

28. Ren, B.; Lin, X. F.; Yang, Z. L.; Liu, G. K.; Aroca, R. F.; Mao B. W.; Tian, Z. Q. SurfaceEnhanced Raman Scattering in the Ultraviolet Spectral Region: UV-SERS on rhodium and ruthenium electrodes. J. Am. Chem. Soc. 2003, 125, 9598-9599.

29. Taylor, C. E.; Pemberton, J. E.; Goodman, G. G.; Schoenfisch, M. H. Surface enhancement factors for $\mathrm{Ag}$ and $\mathrm{Au}$ surfaces relative to $\mathrm{Pt}$ surfaces for monolayers of thiophenol. Appl. Spectrosc. 1999, 53, 1212-1221.

30. Pieczonka, N. P. W.; Aroca, R. F. Inherent complexities of trace detection by surface-enhanced Raman scattering. Chem. Phys. Chem. 2005, 6, 2473-2484.

31. Clarkson, J.; Sudworth, C.; Masca, S. I.; Batchelder, D. N.; Smith, D. A. Ultraviolet resonance Raman study of the avidin biotin complex. J. Raman. Spectrosc. 2000, 31, 373-375.

32. Wang, L.; Yan, R.; Huo, Z.; Wang L.; Zeng J.; Bao, J.; Wang, X.; Peng, Q.; Li, Y. Fluorescence resonant energytransfer biosensor based on upconversion-luminescent nanoparticles. Angew. Chem. Inter. Ed. 2005, 44, 6054-6057. 
33. Dong, D.; Zheng, Dong.; Wang, F.-Q.; Yang, X.-Q.; Wang, N.; Li, Y.-G.; Guo, L.-H.; Cheng, J. Quantitative Photoelectrochemical Detection of Biological Affinity Reaction:Biotin-Avidin Interaction. Anal. Chem. 2004, 76, 499-501.

34. Christophe, L.; Nicolas, H.; Thomas, R. W. Artificial metalloenzymes based on biotin-avidin technology for the enantioselective reduction of ketones by transfer hydrogenation. PNAS 2005, 102, 4683-4687.

35. Dimitrijevic, N. M..; Saponjic, Z. V.; Rabatic, B. M.; Rajh, T. Assembly and charge transfer in hybrid $\mathrm{TiO}_{2}$ architectures using biotin-avidin as a connector. J. Am. Chem. Soc. 2005, 127, 13441345.

36. Torreggiani, A.; Fini, G. Raman spectroscopic studies of ligand-protein interactions: the binding of biotin analogues by avidin. J. Raman. Spectrosc. 1998, 29, 229-236.

37. Torreggiani, A.; Fagnano, C.; Fini, G. Involvement of lysine and tryptophan side-chains in the biotin-avidin interaction. J. Raman. Spectrosc. 1997, 28, 23-27.

38. Honzatko, R. B.; Williams, R. W. Raman spectroscopy of avidin: secondary structure, disulfide conformation, and the environment of tyrosine. Biochem. 1982, 21, 6201-6205.

(C) 2007 by MDPI (http://www.mdpi.org). Reproduction is permitted for noncommercial purposes. 